\title{
Erratum: Quantized conductance through a dissipative atomic point contact [Phys. Rev. A 100, 053605 (2019)]
}

\author{
Laura Corman @, Philipp Fabritius, Samuel Häusler, Jeffrey Mohan ® , Lena H. Dogra, \\ Dominik Husmann, Martin Lebrat, and Tilman Esslinger
}

(Received 18 May 2021; published 28 May 2021)

DOI: 10.1103/PhysRevA.103.059902

In our original article, the linewidths of the resonances in the atomic polarizability $\alpha_{g}^{q q^{\prime}}$ of a particular ground state $|g\rangle$ were erroneously given by the decay rate $\Gamma_{e g}$ from the excited state $|e\rangle$ of the transition to $|g\rangle$. This led to the incorrect conclusion that the small $m_{J}=1 / 2$ component of $|\downarrow\rangle$ gives the eigenstate a narrow resonance shown in Figs. 2(c) and 2(d) that can be exploited to achieve the same spin-dependent potential $V_{s}$ with a lower photon scattering rate. In fact, the linewidth is set by the inverse lifetime of the excited state $\Gamma_{e}$ which can be larger than $\Gamma_{e g}$ due to coupling to other ground states, while only the strength of the transition is set by $\Gamma_{e g}[1]$. With this correction, the polarizability is

$$
\alpha_{g}^{q q^{\prime}}=-\sum_{e} \frac{\left(d_{e g}^{q}\right)^{*} d_{e g}^{q^{\prime}}}{h\left(v-v_{e g}\right)+i \hbar \Gamma_{e} / 2}
$$

where $\Gamma_{e}=\sum_{g} \Gamma_{e g}$. Figure 1 here shows the same properties as in Fig. 2 of our original article with the corrected polarizability, showing that the width of the resonance in Figs. 2(c) and 2(d) is the same as in Figs. 2(a) and 2(b) while its strength is suppressed. Additionally, there are small shifts in the resonance centers as we now use more recent experimental and theoretical values for

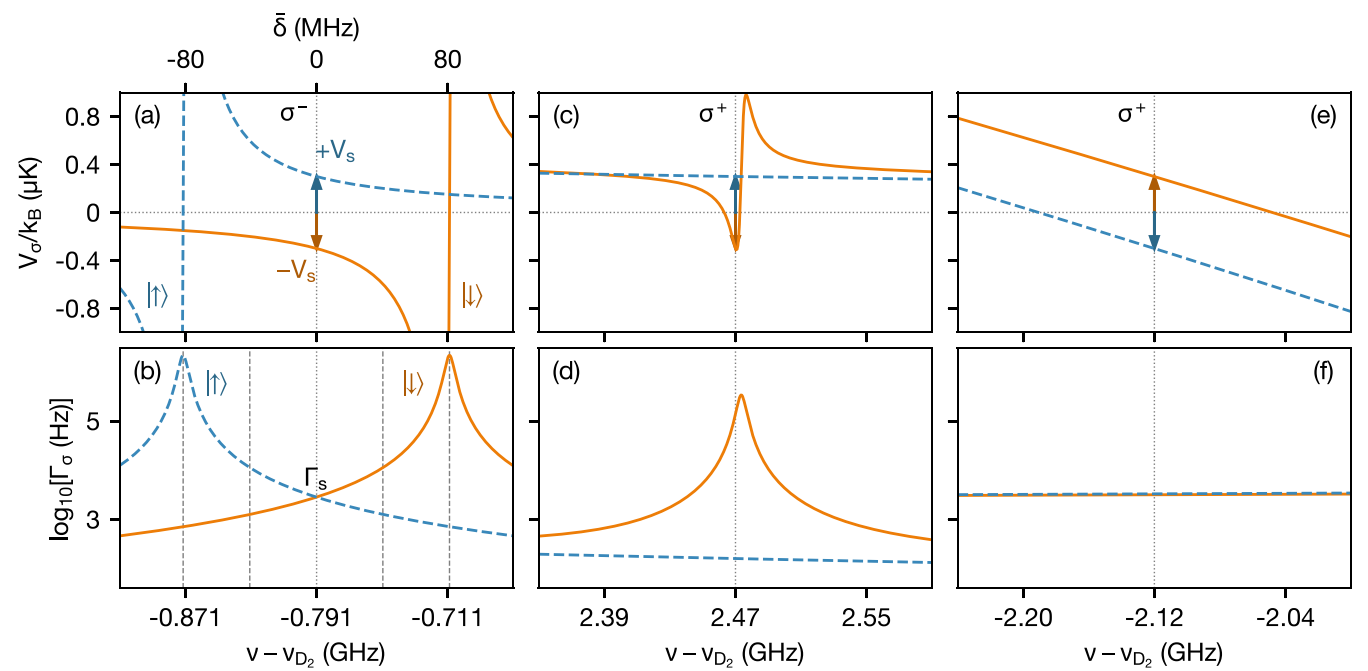

FIG. 1. Potentials and scattering rates at a magnetic field of $B=568 \mathrm{G}$ for the $|\downarrow\rangle$ and $|\uparrow\rangle$ states for different frequencies $v$ of the light where the mean potential $\left(V_{\downarrow}+V_{\uparrow}\right) / 2$ vanishes. The lower frequency axes are offset by the $D_{2}$ transition frequency at zero magnetic field $v_{D_{2}}$ [4]. The intensities in each pair of graphs are chosen to give a constant amplitude of the spin potential $\left|V_{\downarrow}-V_{\uparrow}\right|=k_{B} \times 0.6 \mu \mathrm{K}$. (a) and (b) correspond to $\sigma^{-}$polarization and an intensity of $3 \mathrm{~W} / \mathrm{m}^{2}$. The top frequency axis has been offset by the mean of the resonance frequencies of state $|\downarrow\rangle$ and $|\uparrow\rangle$ for the main $D_{2} \sigma^{-}$transition: $\bar{\delta}=v-\left(v_{\downarrow}+v_{\uparrow}\right) / 2$. (c) and (d) correspond to $\sigma^{+}$polarization and an intensity of $111 \mathrm{~W} / \mathrm{m}^{2}$ close to a weak $D_{2}$ resonance appearing in the Paschen-Back regime. (e) and (f) correspond to $\sigma^{+}$polarization and an intensity of $1.2 \times$ $10^{4} \mathrm{~W} / \mathrm{m}^{2}$ between the $D_{1}$ and $D_{2}$ lines. 
the hyperfine coupling constants of the $S_{1 / 2}$ and $P_{1 / 2}$ manifolds [2], the hyperfine coupling constant of the $P_{3 / 2}$ manifold [3,4], fine-structure splitting [4], and nuclear and electronic $g$ factors [3].

[1] F. Le Kien, P. Schneeweiss, and A. Rauschenbeutel, Eur. Phys. J. D 67, 92 (2013).

[2] C. J. Sansonetti, C. E. Simien, J. D. Gillaspy, J. N. Tan, S. M. Brewer, R. C. Brown, S. Wu, and J. V. Porto, Phys. Rev. Lett. 107, 023001 (2011).
[3] M. Puchalski and K. Pachucki, Phys. Rev. A 79, 032510 (2009).

[4] R. C. Brown, S. Wu, J. V. Porto, C. J. Sansonetti, C. E. Simien, S. M. Brewer, J. N. Tan, and J. D. Gillaspy, Phys. Rev. A 87, 032504 (2013). 